\title{
A LOW COST SYNTHESIS AND CHARACTERIZATION OF CUO NANOPARTICLES FOR PHOTOVOLTAIC APPLICATIONS
}

\author{
A. A. Yankson*, A. Kuditcher, G. Gebreyesus, M. N. Y. H. Egblewogbe, \\ J. K. A. Amuzu And E. A. Armah \\ (Department of Physics, University of Ghana, Legon). \\ *Corresponding author's email: ayankson@ug.edu.gh
}

\begin{abstract}
A simple low cost chemical route has been used to synthesize cupric oxide nanoparticles. The synthesized $\mathrm{CuO}$ nanoparticles were characterized using XRD, TEM and UV-absorption. $\mathrm{X}$-ray diffraction analysis showed the synthesized nanoparticles to be a pure cupric oxide. EDAX analysis showed the presence of copper and Oxygen in the as prepared $\mathrm{CuO}$ nano particles, with the AAS indicating that $\mathrm{Cu}^{2+}$ represented $53.5 \%$ of the sample. The particle size and particle size distribution of the cupric oxide nanoparticles were obtained by transmission electron microscopy (TEM) whereas the crystallite size and crystallite size distribution were obtained by X-ray diffraction. The particle size was found to be between $20 \mathrm{~nm}$ and $60 \mathrm{~nm}$. The particle size distribution obtained from cumulative percentage frequency plots features a log-normal function. Absorbance measurements and analysis showed that the material has an absorbance peak at $314 \mathrm{~nm}$ and energy bandgap of $1.48 \mathrm{eV}$, making it a good candidate for photovoltaic applications.
\end{abstract}

Keywords: $\mathrm{CuO}$, inexpensive chemical route, particle size, crystallite size, absorbance peak, band gap

\section{Introduction}

Transition metal oxide nanoparticles are of great importance due to their application in the field of sensing, optoelectronics, catalysis and photovoltaics which hinge on their unique physical and chemical properties differing from the bulk (Selvi et al., 2016) (Swankar et al., 2009) (Srivastra et al., 2013). Cupric oxide nanoparticles have attracted a lot of attention lately due to their unique properties. Cupric oxide is a p-type semiconductor with an indirect narrow band gap of $1.21-1.51 \mathrm{eV}$ and it has a monoclinic structure (Armelao et al., 2003). Besides its wide use in power sources such as in photovoltaic devices, it is suitable for use as an electrode in lithium batteries (Ezenwa, 2012) (Suleiman et al., 2013). The use of photovoltaic modules in areas of high insolation requires that solar cells are made of materials that do not degrade at high operating temperatures. Some inorganic materials including cupric oxide are known not to degrade at high temperatures, making cupric oxide suitable for solar cell fabrication. The cost of solar cells is an important factor in the production and affordability of solar cells and synthesis of materials for the fabrication of solar cells should not be expensive (Kidowaki et al., 2012). The synthesis approaches of $\mathrm{CuO}$ nanoparticles have advanced essentially over the past years because of their notable 
biomedical and industrial approaches (Singh et al., 2016). In this paper we report a simple low cost chemical route for the synthesis of cupric oxide nanoparticles.

\section{Materials}

\section{Experimental}

Analytical grade copper sulphate tetrahydrate and $30 \%$ ammonia water were used as the precursors. De-ionised water was used as solvent.

\section{Method}

The appropriate molar concentrations of the reagents were prepared in de-ionized water. $7.48806 \mathrm{~g}$ of $\mathrm{CuSO}_{4} \cdot 5 \mathrm{H}_{2} \mathrm{O}$ was added to 300 $\mathrm{ml}$ of de-ionized water to produce $0.1 \mathrm{M}$ concentration of the hydrated salt. $100 \mathrm{ml}$ of it was added to $20 \mathrm{ml}$ of $30 \%$ ammonium solution and the mixture stirred vigorously with a magnetic stirrer. This resulted in a deep blue solution which was kept at $80^{\circ} \mathrm{C}$ for 45 minutes during which time a black precipitate was observed with colourless filtrate. The colourless supernatant was poured out of the beaker and the precipitate washed with excess de-ionized water five times to ensure that no trace of the reagents remained. The precipitate was dried on a hot plate at $50^{\circ} \mathrm{C}$ for 2 hours. A fine black powder was obtained.

The powder was prepared for $\mathrm{x}$-ray diffraction (XRD) analysis, optical analysis using a UV-VIS absorption spectrometer, particle size analysis using transmission electron microscopy (TEM), morphology and elemental analysis by scanning electron microscopy (SEM) and energy dispersive $\mathrm{X}$-ray spectroscopy (EDX). Atomic absorption spectroscopy (AAS) was used to determine the presence of $\mathrm{Cu}^{2+}$ in the as prepared sample.

\section{Results and Discussion}

The powder was analysed using X-ray powder diffraction (XRD) measurements on a the Panalytical Empyrean with $\mathrm{Cu} \mathrm{K}_{\alpha}$ radiation at over 10 - $902 \theta$ degrees. Five samples were prepared using the same method, and the X-ray measurements showed that they were all $\mathrm{CuO}$ nanoparticles. The XRD was used to obtain the crystallite size by applying the Scherrer equation. The resulting powder was kept for six months to investigate whether or not there would be a colour change in the powder. No colour change was observed.

Fig. 1 shows the XRD pattern of as synthesized cupric oxide nanoparticles. The XRD data was analysed using the Panalytical High Score software. Table 1 provides the following information from the XRD data on the as prepared cupric oxide nanoparticle. These are

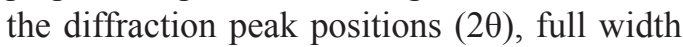
at half maximum (FWHM) and d-spacing related to the individual diffraction peaks. The grain or crystallite sizes for the various FWHM values are also shown in the table. The XRD data showed that the prepared powders are cupric oxide nanoparticles. The Scherrer Equation (Equation 1) was used to calculate an approximate value for the crystallite size.

Where, $\lambda$ is the wavelength of the $\mathrm{x}$-rays used, $\beta$ is the full wave at half maximum of the selected $\mathrm{x}$-ray reflection peak, $k$ is the shape factor with a value of 0.9 (Suleiman et al., 2013). The Scherrer equation yielded crystallite size in the range $13.1 \mathrm{~nm}-27.6 \mathrm{~nm}$, with an average size of $18.4 \mathrm{~nm}$. 


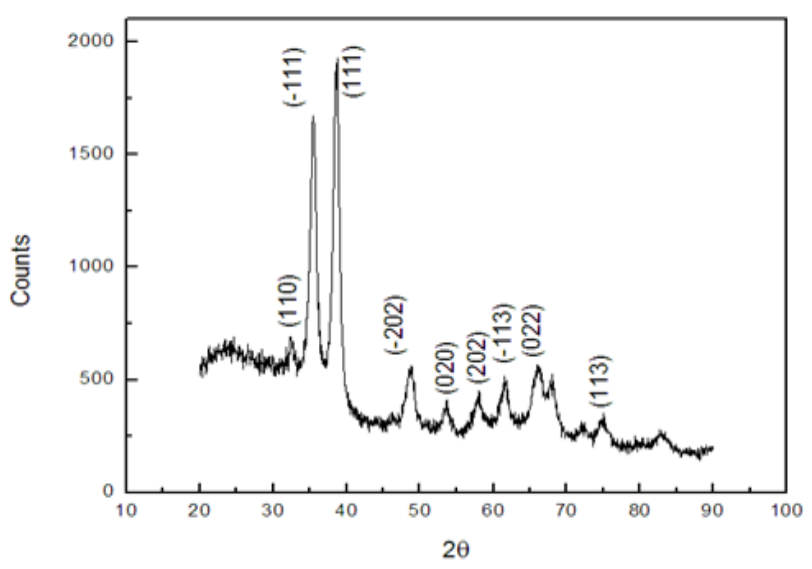

Fig. 1: XRD pattern of as synthesized cupric oxide nanoparticles. The Miller indices of the crystal planes are indicated in the parenthesis with their corresponding peaks.

TABLE 1

Parameters obtained from XRD measurements

\begin{tabular}{|c|c|c|c|c|}
\hline Postion[20] & Height [cts] & FWHM Left[2 $\theta]$ & d-spacing $[\AA]$ & Rel. Int. [\%] \\
\hline 35.4856 & $6097(47)$ & 0.3444 & 2.52769 & 97.45 \\
\hline 38.6838 & $6257(36)$ & 0.6396 & 2.32575 & 100.00 \\
\hline 48.8764 & $825(17)$ & 0.8856 & 1.86192 & 13.19 \\
\hline 53.5889 & 294(18) & 0.5904 & 1.70877 & 4.69 \\
\hline 58.2309 & $414(16)$ & 0.7872 & 1.58312 & 6.62 \\
\hline 61.4033 & $904(20)$ & 0.5904 & 1.50871 & 14.45 \\
\hline 66.0648 & $1171(17)$ & 0.9840 & 1.41310 & 18.71 \\
\hline 68.2960 & $630(17)$ & 0.7872 & 1.37227 & 10.07 \\
\hline 72.1657 & 0.9840 & 0.9840 & 1.30791 & 0.36 \\
\hline
\end{tabular}



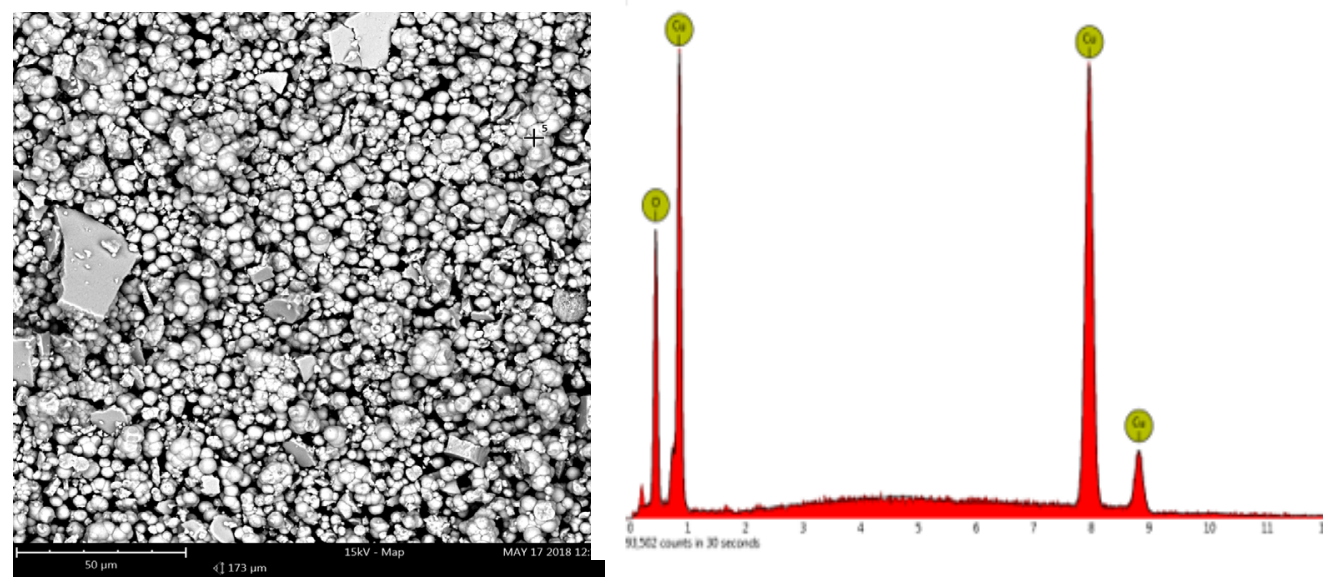

Fig. 2: SEM and EDAX images of CuO: EDAX is very important in identifying elements at the nano scale (Scimeca et al., 2018).

Fig. 3 shows one of the micrographs prepared from TEM images which were analyzed using an optical microscope to determine the particle size distribution. These measurements were tallied and a histogram plotted for the determination of size distribution of the asprepared $\mathrm{CuO}$ nanoparticles.

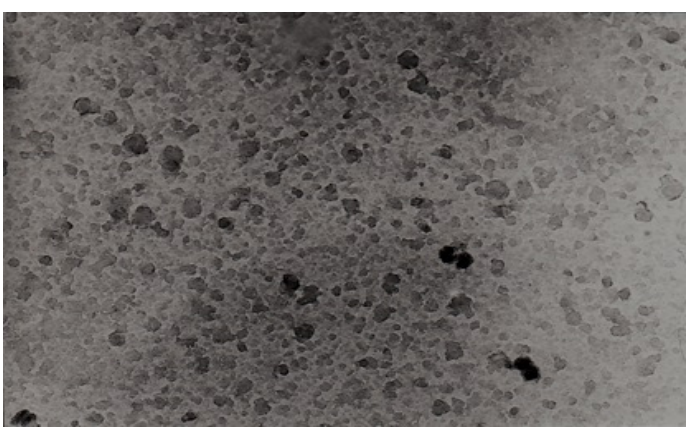

Fig. 3: Micrograph of $\mathrm{CuO}$ nanoparticles $[\times 50,000]$ The particle size distribution data obtained from the micrograph, counting more than 500 particles have been presented in both tabular and graphical form.
TABLE 2

The particle size data extracted from the TEM micrograph

\begin{tabular}{ll}
\hline Mean $(\mathrm{nm})$ & 52.2 \\
\hline Median $(\mathrm{nm})$ & 25.0 \\
\hline Mode $(\mathrm{nm})$ & 22.2 \\
\hline Standard deviation & 11.1 \\
\hline Range $(\mathrm{nm})$ & 50.0 \\
\hline Minimum $(\mathrm{nm})$ & 5.6 \\
\hline Maximum $(\mathrm{nm})$ & 55.6 \\
\hline Count & 522 \\
\hline
\end{tabular}

A number frequency histogram of the particle size data in a linear scale has been plotted. The histogram can give a valid size frequency curve drawn through it if and only if hundreds of particles say between 500 and 1000 are measured to give statistically useful mean size data. (Wang et al., 2012) (Ezenwa, 2012). 


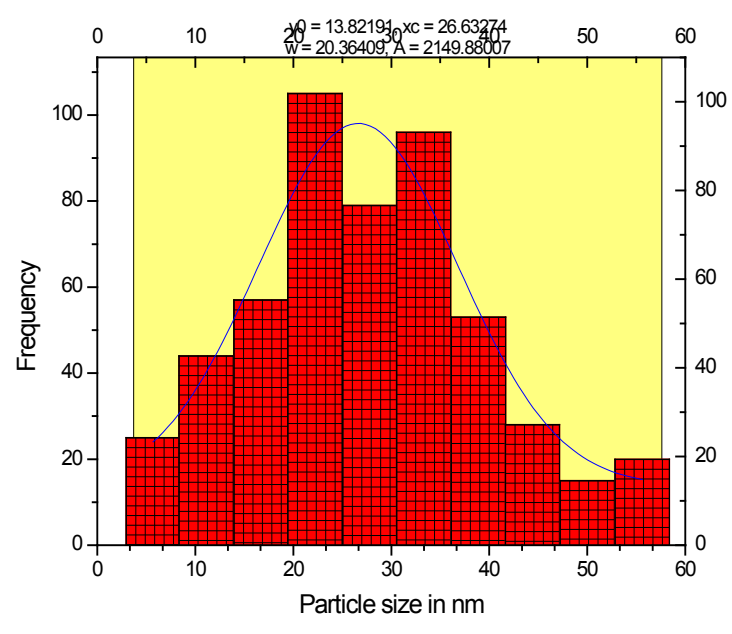

Fig. 4: Number frequency histograms showing particle size distribution with a Gaussian fit line. The particle size data is collected from image analysis of more than 500 particles.

From the histogram in Fig.4, the particle size distribution is approximately even over the entire selected size population. The mean particle size as obtained for the histogram is $52.20 \mathrm{~nm}$ with a standard deviation of 11.1. The histogram reveals the particle size range.

\section{Band gap measurement}

UV-visible absorption of the as synthesized cupric oxide nanoparticles were recorded in the visible to near infra- red region. The spectrum has one peak centered around $314 \mathrm{~nm}$, which indicates that the as synthesized cupric oxide nanoparticles absorbs in the ultra violet region, a good optical property of a material that is needed for photovoltaic applications.

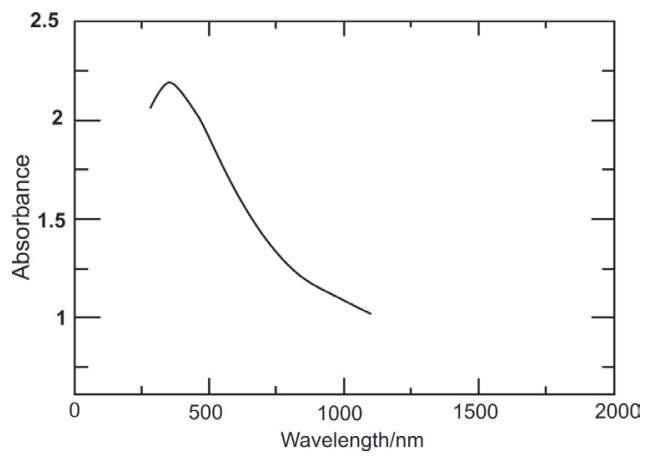

Fig. 5: UV-VIS absorption spectrum of as synthesized cupric oxide nanoparticles.

The band gap energy of a given material is an important characteristic for a semiconductor material. The band gap energy of the as synthesized cupric oxide was determined with the help of the Tauc's relation (Tauc, 1974). This has been achieved by using several absorption measurements and the mean value obtained for the band gap.

From Tauc's equation where is the absorption coefficient, is the frequency of the incident photon, $h$ is Planck's constant and $n$ is 0.5 or 2 for direct and indirect transitions respectively. A Tauc plot, versus, which provides the best linear relationship for direct transition, was used to obtain the optical band gap as shown in Fig. 6.

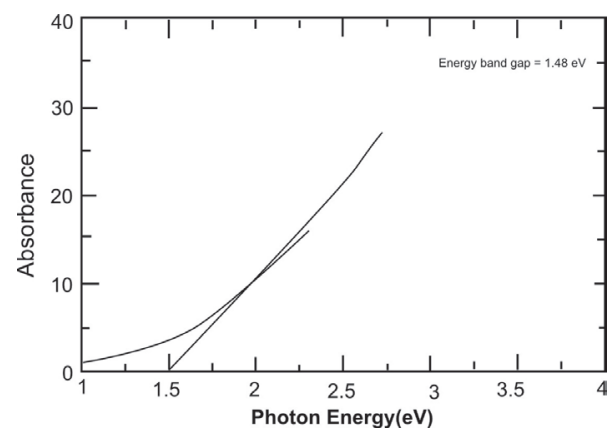

Fig. 6: Absorption spectrum ( versus ) for Tauc plot calculation 
The numerical value of the band gap is obtained from the straight line drawn from the most linear part of the curve cutting the axis, where is zero. The mean optical band gap was calculated to be $1.48 \pm 0.04 \mathrm{eV}$. This band gap value is higher than the $1.37 \mathrm{eV}$ obtained [Ooi et al., 2014] but lower than band gap values obtained by (Pierson et al., 2003) and (Ogwu et al., 2007) which were 2.11 and $2.05 \mathrm{eV}$ respectively.

It also falls within the range of value for the bulk $\mathrm{CuO}$, from $1.0-1.5 \mathrm{eV}$ but is less than the reported value of $2.4 \mathrm{eV}$ (Johan et al., 2011).

\section{Conclusion}

This work has successfully produced black cupric oxide nanoparticles with suitable properties an indication of its potential for photovoltaic applications from a simple low cost chemical route. The XRD analyses has shown that the black powder prepared has $100 \% \mathrm{CuO}$ phase with the two highest peaks occurring at $35.5^{\circ}(2 \theta)$ and $38.7^{\circ} 2(\theta)$ corresponding to reflections from the and 111 atomic planes of the crystallites. The AAS analysis indicated that there was $53.5 \% \mathrm{Cu}^{2+}$ present in the as prepared $\mathrm{CuO}$ nano particles and EDAX analysis confirming the presence of copper and oxygen in the $\mathrm{CuO}$ nanoparticles. From the XRD results and subsequent calculations, the average crystallite size ranges from $8.43 \mathrm{~nm}-25.29 \mathrm{~nm}$.

Samples of the prepared cupric oxide nanoparticles have average particle size range $20.0 \mathrm{~nm}-60.0 \mathrm{~nm}$, with the size distribution spreading almost evenly around the mean particle size on the particle size scale as shown by the analysis of micrographs from TEM measurements. Both TEM and SEM images showed that the as prepared $\mathrm{CuO}$ nano particles were largely spherical.
The sample absorbed in the visible and ultra violet region peaking around $314 \mathrm{~nm}$, making it a good candidate for photovoltaic applications. The as synthesized cupric oxide has a mean optical bandgap energy of $1.48 \mathrm{eV} \pm 0.05$ $\mathrm{eV}$ which falls within the range of bandgap energies suitable for photovoltaic applications.

\section{Acknowledgments}

The authors are grateful to the UG-Carnegie NGAA Project for supporting the work financially.

\section{References}

Armelao, L., Barreca, D., Bertapella, M., et al., (2003) A sol-gel approach to nanophasic copper oxide thin films. Thin Solid Films 442, 48 -52 .

Ezenwa, I. A. (2012) Optical Analysis of Chemical bath Fabricated $\mathrm{CuO}$ Thin Films. Res. J.Recent. Sci. 1 (1), 46 - 50.

Johan M. R., Suan M. S. M., Hawari N. L., et al., (2011) Annealing effects on the properties of copper oxide films prepared by chemical deposition, Int. J. Electrochem. Sci. 6, 6094 $-6104$.

Kidowaki, H., Oku, T., Akiyama, T., et al., (2012) Fabrication and Characterization of CuO-based Solar Cells. JMSR 1 (1), 138.

Ogwu, A., Darma, T. \& Bouquerel, E. (2007) Electrical resistivity of copper oxide thin films prepared by reactive magnetron sputtering. JAMME 24 (1).

OoI, P. K. \& Ching, C. G. \& Ahmad, M., et al., (2014) Characterizations of Cupric Oxide thin Films on Glass and Silicon Substrates by Radio Frequency Magnetron Sputtering, Sains Malaysiana 43 (4), 617 - 621.

Pierson, J. F., Thobor-Keck, A. \& Billard, A. (2003) Cuprite, paramelaconite and teronite films de- 
posited by reactive magnetron sputtering. $A p$ plied Surface Science 210 (3 - 4), 359 - 367.

Scimeca, M., Bischetti, S. \& Kaur L., et al., (2018) Energy dispersive X-ray (EDX)microanalysis: A powerful tool in biomedical research and diagnosis. European Journal of Histochemical 62 (1), 2841.

Selvi K, T., Alamelumangi, K., Rathnakumari, M., et al., (2016) Synthesis and Study of Optical properties MgObased $\mathrm{TM}$ oxide $(\mathrm{TM}=\mathrm{Cu}$, $\mathrm{Mn}$ and $\mathrm{Zn}$ ) nanocomposites. Mater. Res. Express 3 115017,

Singh, J., Kaur, G. \& Rawat, M. A. (2016) A Brief Review on Synthesis and Characterization of Copper Oxide Nanoparticles and its Applications. J. Bioelectron. Nanotechnol. 1 (1), $1-9$.

Srivastava, S., Kumar, M., Agrawal, A., et al., (2013) Synthesis and Characterization of Copper Oxide nanoparticles. ISOR-JAP 5 (4), 61 - 65.
Suleiman, Mousa M., Hussein A., et al., (2013) Copper(II)-Oxide Nanostructures: Synthesis, Characterizations and their Applications-Review. J. Mater. Environ. Sci. 4 (5), $792-797$.

Swankar, R. K., Singh, S. C. \& Gopal, R. (2009) Optical Charactrization of Copper Oxide Nanomaterial. ICOP-Intrnational Conference on Optics and Photonics CSIO,Chandigarh, India.

TAuC, J. (1974) “Amorphous and liquid semiconductors" Plenum, New York, 159.

Wang, T. X., Xu, S. H. \& Yang F. X. (2012) Green synthesis of $\mathrm{CuO}$ nanoflakes from $\mathrm{CuCO}_{3}$. $\mathrm{Cu}(\mathrm{OH})_{2}$ powder and $\mathrm{H}_{2} \mathrm{O}_{2}$ aqueous solution, Powder Technology 228, 128 - 130.

Received 27 Jan 19; revised 26 Jul 19. 